\title{
Coal and fuel burning effects on the atmosphere as mediated by the atmospheric electric field and galactic cosmic rays flux
}

\author{
A. Heitor Reis* \\ Geophysics Centre of Évora \\ Rua Romão Ramalho 59 \\ 7000-671 Évora, Portugal \\ and \\ Department of Physics \\ University of Évora \\ Apartado 94, 7002-554 Évora, Portugal \\ E-mail: ahr@uevora.pt \\ *Corresponding author

\section{Cláudia Serrano} \\ Geophysics Centre of Évora \\ Rua Romão Ramalho 59 \\ 7000-671 Évora, Portugal \\ E-mail: claudiafserrano@yahoo.com.br
}

\begin{abstract}
Emissions into the atmosphere of Greenhouse Gases (GHGs) and particulate matter resulting from fossil fuel burning are considered to be the main anthropogenic forcing on the global climate. We show here that the external cyclic influences of cosmic origin that modulate the earth's climate may either reinforce or mitigate the 'local' terrestrial forcings. Among the external influences is cosmic radiation, whose intensity shows a cyclic variation of 11 years, accompanying the 11-year cycle of solar activity. We put forward a mechanism to explain how the emission of particulate matter into the atmosphere might influence global lightning activity. With respect to global lightning activity, we show why, during the 11-year cycle, the influence of an increase in particulate matter concentration in the atmosphere may be negligible in some years, while it will be reinforced in other years, depending on the place of the years in the cycle. We also remark that the effect on global warming of fossil fuel burning is also modulated by the cosmic ray flux, whose influence is mediated by the variation that it promotes on the cloud cover.
\end{abstract}

Keywords: coal; fuel; global warming; atmosphere; electric field.

Reference to this paper should be made as follows: Heitor Reis, A. and Serrano, C. (2009) 'Coal and fuel burning effects on the atmosphere as mediated by the atmospheric electric field and galactic cosmic rays flux', Int. J. Global Warming, Vol. 1, Nos. 1/2/3, pp.57-65.

Biographical notes: Antonio Heitor Reis graduated with a degree in Physics from the University of Lisbon and received his MSc in Mechanical Engineering from the Technical University of Lisbon (IST) and his PhD in Physics from the University of Évora, Portugal. From 1981 to 1986, he did research on energy at 
the National Laboratory for Engineering and Technology. He joined the University of Évora in 1986, where he teaches Physics and Engineering courses. He is currently the Vice Rector for Research and Technology at the University of Évora. His current research interests concern energy issues, atmospheric physics, flows in porous media (animate and inanimate) and constructal theory.

Cláudia Serrano is a PhD student at the University of Évora, Portugal. Her research work is focused on the planetary and local influences on the atmospheric electric field at ground level.

\section{Introduction}

Global changes in the temperature of Earth's atmosphere are due to multiple influences. Long-term influences are of astronomical origin, namely the eccentricity, axial tilt and precession of the Earth's orbit, and are thought to be responsible for climate changes for periods of the order $10^{5}$ years (Milankovic cycles). Short-term climate changes with periods of order $10-10^{2}$ years are due to:

- external influences like solar activity that modulate the Galactic Cosmic Rays (GCR) flux, and changes in GCRs that reach the solar system

- Anthropogenic Factors (AFs) such as fossil fuels burning at increasing high rates that increase Greenhouse Gases (GHGs) in the atmosphere (carbon dioxide, methane, etc.) as well as aerosol concentration, and Natural Factors (NFs) like volcanic eruptions that increase particle concentration in the atmosphere.

In the atmosphere, the increase in particulate matter concentration resulting from fossil fuel burning and other sources contributes to enhancing the cloud cover, which combined with the direct effect of light reflection by aerosols increases the albedo of the earth. As a consequence, less solar radiation is absorbed at the surface.

On the other hand, the increasing concentration of GHGs has the effect of impeding the terrestrial radiation's escape into space, thus increasing the greenhouse factor. The major contributor is carbon dioxide, whose main anthropogenic source is fossil fuel burning. Methane, nitrous oxide and halocarbons are other important GHGs of anthropogenic origin. The trade-off between these opposite trends generates as a global result an actual positive radiative imbalance of order $0.85 \mathrm{~W} / \mathrm{m}^{2}$ (Hansen et al., 2005).

In this work we address the role of the emissions into the atmosphere of gases and particulate matter resulting from coal burning and fuel burning. Because particulate matter has an overall cooling effect while gases increase the greenhouse effect, there is a trade-off that generally results in a net increase in temperature.

\section{Current evidence of global warming}

Evidence of a rapid rise in global temperature in the last decades is accumulating. The 2007 Report of the Intergovernmental Panel in Climate Change (IPPC Report, 2007) shows that global warming is a fact, the marks of which can be seen in many earth 
systems. Examples are everywhere, e.g., enlargement and increased number of glacial lakes, increasing ground instabilities in permafrost regions, changes in Arctic and Antarctic ecosystems, and glacier retraction and reduced extensions of ice-covered regions. The effects on high-latitude forests, earlier plantation of crops, spreading of pests and changes in infectious disease vectors in Europe have also been documented.

As remarked in the IPPC Report, the average surface temperature and sea level have been increasing consistently during the second half of the 20th century. In contrast, the snow cover in the Northern Hemisphere has decreased reduced during the same period.

GHGs have been considered the major drivers of global warming. In the period 1970-2004, global GHG emissions of anthropogenic origin increased by $70 \%$. In the same period, carbon dioxide emissions increased from 21 to 38 gigatonnes and represented $77 \%$ of GHG emissions in 2004 , the major part of which was due to energy plants, transportation and industry (IPPC Report, 2007). As a consequence of the GHG emissions, GHG concentrations in the atmosphere have increased in relation to those of the pre-industrial era. Carbon dioxide concentration increased from $280 \mathrm{ppm}$ to $379 \mathrm{ppm}$ (2005), while concentrations of methane and nitrous oxide increased from $715 \mathrm{ppb}$ to $1732 \mathrm{ppb}$ (2005) and from $270 \mathrm{ppb}$ to $319 \mathrm{ppb}$ (2005), respectively (IPPC Report, 2007).

The direct contribution of carbon dioxide to radiative forcing is estimated to be of order $1.66 \mathrm{~W} / \mathrm{m}^{2}$, while those of methane, nitrous oxide and halocarbons is of order $0.48 \mathrm{~W} / \mathrm{m}^{2}, 0.16 \mathrm{~W} / \mathrm{m}^{2}$ and $0.34 \mathrm{~W} / \mathrm{m}^{2}$, respectively. Trace gases also give a contribution to radiative forcing. The direct and indirect effects of particulate matter on radiative forcing are found to be negative and of order $-0.12 \mathrm{~W} / \mathrm{m}^{2}$ (IPPC Report, 2007). The fact that GHG concentrations are positively correlated with global temperature has been clearly demonstrated by the measurements in the Vostok ice core, which evinced that the variations in carbon dioxide and methane concentrations in the atmosphere matched the temperature variations closely (Hansen and Sato, 2004).

This scenario challenges the current energy paradigm and points to the need of reducing global GHG emissions. Though global changes in conventional energy supply systems are not easy to achieve, some policies must be globally implemented in the short run. The Kyoto Protocol (1998) and the recent Bali Agreement (2007) represent global efforts to put forward a global strategy for the reduction of GHG emissions.

\section{The atmospheric electric field and electric power in the atmosphere}

Ions exist in variable concentrations in the atmosphere. Among the many existing ion sources, the most important are solar radiation, natural and artificial radioactivity and cosmic rays. Because charge concentration is not equally distributed in the atmosphere, electric fields develop that can reach magnitudes of order tenths of kilovolts per metre during thunderstorms. In fair weather conditions the average atmospheric electric field at ground level is of order 100-150 V/m, and points vertically downward.

The simplest model usually accepted for describing the electric state of the atmosphere is that of the spherical capacitor. According to this model, the ionosphere is positively charged while the earth's surface is negatively charged. A current of order $2 \mathrm{pA} / \mathrm{m}^{2}$ is established between the ionosphere and the earth's surface. In fair-weather conditions this current consists of positive ions that flow towards the ground. In stormy 
conditions, energetic electric discharges (lightning) occur between the atmosphere and the ground, and vice versa. The capacitor is continuously being charged by thunderstorms occurring throughout the globe. This model is very useful for understanding atmospheric electricity because it captures and describes the essence of the electric phenomenology in the atmosphere.

In fair weather, the atmospheric electric field $E_{z}$ induces a current density $J_{z}$ that is almost ohmic and is given by:

$$
\mathrm{J}_{0}=\sigma \mathrm{E},
$$

where $\sigma$ is the electric conductivity of the air (see, for example, McGorman and Rust, 1998). As the fair-weather current density is nearly constant, the field strength varies inversely with the electric conductivity of the air, which depends on the concentration and mobility of the atmospheric ions. The electric conductivity is related to the ion concentration $\mathrm{n}$, electric charge $\mathrm{q}$ and mobility $\mu$, through:

$$
\sigma=\mathrm{n}_{+} \mathrm{q}_{+} \mu_{+}+\mathrm{n}_{-} \mathrm{q}_{-} \mu_{-}
$$

where the subscripts + and - refer to positive and negative charges, respectively.

Because the average current density does not change much, from Equations (1-2) we conclude that the product of ion density and ion mobility practically determines the fair-weather electric field strength. Ion mobility is strongly reduced if small droplets grow hygroscopically from existing ions or if ions are captured by droplets or by particulate matter in the atmosphere.

In general, the current $\mathrm{J}$ that flows between the ionosphere and the ground is composed of the ohmic current plus the discharge current $\mathrm{J}_{\mathrm{L}}$, which is given by $(\varepsilon$ is electric permittivity):

$$
\mathrm{J}_{\mathrm{L}}=\varepsilon \partial \mathrm{E} / \partial \mathrm{t}
$$

The electric power intensity, $\mathrm{W}_{\mathrm{E}}\left(\mathrm{W} / \mathrm{m}^{2}\right)$, carried by this global current $\mathrm{J}=\mathrm{J}_{\mathrm{z}}+\mathrm{J}_{\mathrm{L}}$ (see Figure 1) is given by $\mathrm{W}_{\mathrm{E}}=\mathrm{JE}$, i.e.:

$$
\mathrm{W}_{\mathrm{E}}=\sigma \mathrm{E}^{2}+\left(\frac{\varepsilon}{2}\right)\left(\frac{\partial \mathrm{E}^{2}}{\partial \mathrm{t}}\right) .
$$

The emission into the atmosphere of particulate matter resulting from fossil fuel burning (or particulate matter formed in the atmosphere from precursor gases resulting from fossil fuel burning) has the effect of reducing ion mobility, due to binding of the existing ions. Additionally, atmospheric particulate matter may act as seeds for droplet nucleation. Droplets are good ion collectors that also contribute to reducing ion mobility. The electric conductivity is reduced accordingly and the same happens with the electric power carried by the ohmic current, as we can conclude by examining the first term in the right-hand side of Equation (4). Because the electric power must be discharged onto the earth's surface, at the first stage the electric field rises accordingly. However, the increase in the electric field occurs only until the air electric breakdown threshold is reached and lightning occurs (the second term in the right-hand side of Equation 4). In this way, the emission of particulate matter from fossil fuel burning must contribute somewhat to the increase in the number of extreme electric events in the atmosphere. 
Figure 1 The two branches of the downward atmospheric electric current $\mathbf{J}$ : the ohmic branch, $\mathbf{J}_{\mathbf{z}}$, and the discharge branch, $\mathbf{J}_{\mathbf{L}}$, corresponding mainly to lightning and corona discharges

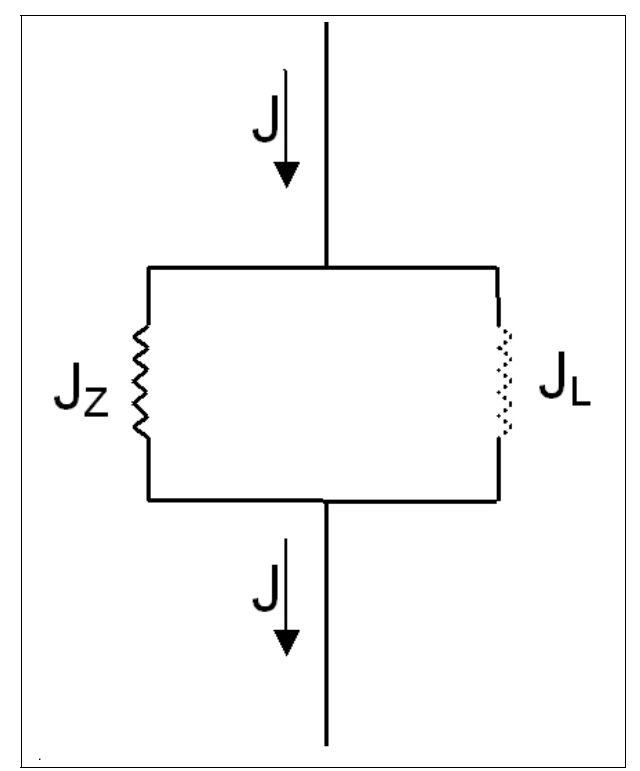

There is some evidence that the effect anticipated by this simple calculation is already active. Markson and Price (1999) showed that the observed increase in global lightning activity is correlated with a temperature increase, though the same might also happen with the aerosol concentration in the atmosphere.

Studies in North America have shown enhancements in cloud-to-ground flashes (Lyons et al., 1998) that have been attributed to incursions of smoke from fires in Mexico and subsequent ingestion by these thunderstorms. On the other hand, subsequent studies of thunderstorms in Brazil formed over smoke from biomass burning (Williams et al., 2002) do not corroborate the results found for North America. However, it is recognised that a global study on the influence of aerosol concentration on the enhancement of lightning activity is still missing.

\section{Combined forcing of particulate matter and cosmic radiation}

Cosmic radiation is the major ionising source in the atmosphere. Cosmic radiation intensity varies with an 11-year period following a known correspondence with solar sunspot activity. The peak of cosmic radiation intensity corresponds to a minimum of the number of solar sunspots, the so-called minimum of the solar cycle. High solar activity through solar wind promotes higher shielding to cosmic rays, and therefore decreasing ionisation in the atmosphere.

Because the ion concentration is directly related to air conductivity (see Equation 2), it is expected that cosmic rays will contribute to the discharge of the atmospheric electric power onto the earth's surface. In fact, due to the amount of intermediate processes occurring in the atmosphere, this effect is not as evident as it would seem to be at first sight. 
While investigating if this effect is real, Serrano et al. (2006), using data series (1955-1991), found that for Lisbon and at the level of statistical significance ( $p$-value $<0,05)$, the atmospheric electric field strength at ground level and cosmic radiation intensity were inversely correlated (see Figure 2).

This means that cosmic radiation somehow modulates the atmospheric electric conductivity and therefore the discharge of electric power from the atmosphere onto the earth's surface.

\subsection{Modulation of lightning activity by cosmic radiation}

In this way, two trends might either reinforce or cancel each other with respect to the electric power available in the atmosphere. The increase in the emission or subsequent generation of particulate matter in the atmosphere as the result of fossil fuel burning seems to increase the electric power in the atmosphere, leading to increasing lightning activity. On the other hand, cosmic radiation promotes ionisation and facilitates discharge onto the ground by the ohmic branch of the circuit. The net effect may be negligible in the years corresponding to the minimum of the solar cycle, i.e., when cosmic radiation is at its maximum and facilitates discharge of power through the ohmic branch. However, when the solar sunspot activity is at its maximum, i.e., when cosmic radiation intensity is close to minimum, the resistance of the ohmic branch is increased and we shall expect increasing lightning activity. This aspect might explain why the correlation between particulate matter concentration and lightning activity was found to be significant for North America but not for Brazil. In fact, the work by Lyons et al. (1998) reports large peak current lightning flashes recorded by the National Lightning Detection Network (NLDN) during 14 summer months in four years, 1991-1995, while that of Williams et al. (2002) refers to the campaign Large-Scale Biosphere-Atmosphere Experiment in Amazonia-Smoke Aerosols (LBA-SMOCC) that occurred in 2002. In 1991-1995 cosmic radiation was close to the minimum (see Figure 2) when it was expected to increase in lightning activity, while during the campaign LBA-SMOCC (2002), cosmic radiation intensity was close to its maximum; therefore according to the reasons explained above, the correlation between lightning activity and aerosols should be negligible, as was found by Williams et al. (2002).

Even though the data on lightning activity from these two campaigns seem to corroborate the explanation proposed here, more data is needed for its confirmation.

\subsection{Modulation of global warming by cosmic radiation}

In a notable paper, Svensmark and Friis-Christensen (1997) report their finding of a correlation between a variation of $3 \%-4 \%$ in global cloud cover and cosmic rays flux during the 11-year solar cycle. That variation was most prominent at higher latitudes in accordance with the shielding effect of the geomagnetic field on cosmic rays. Moreover, the cooling effect resulting from the $3 \%-4 \%$ variation in cloud cover was estimated from satellite measurements to be in the range $0.8-1.7 \mathrm{~W} / \mathrm{m}^{2}$. This value is of the same order as the current positive radiative imbalance of $0.85 \mathrm{~W} / \mathrm{m}^{2}$ found by Hansen et al. (2005), and deserves scrutiny. Actually, the 11-year period cosmic ray effect may either reinforce or mitigate the global warming effect resulting from fossil fuel burning. 
Figure 2 (a) The vertical component of the atmospheric electric field in Lisbon and (b) the normalised variation of cosmic radiation intensity in various stations, which is practically independent of the latitude (see online version for colours)

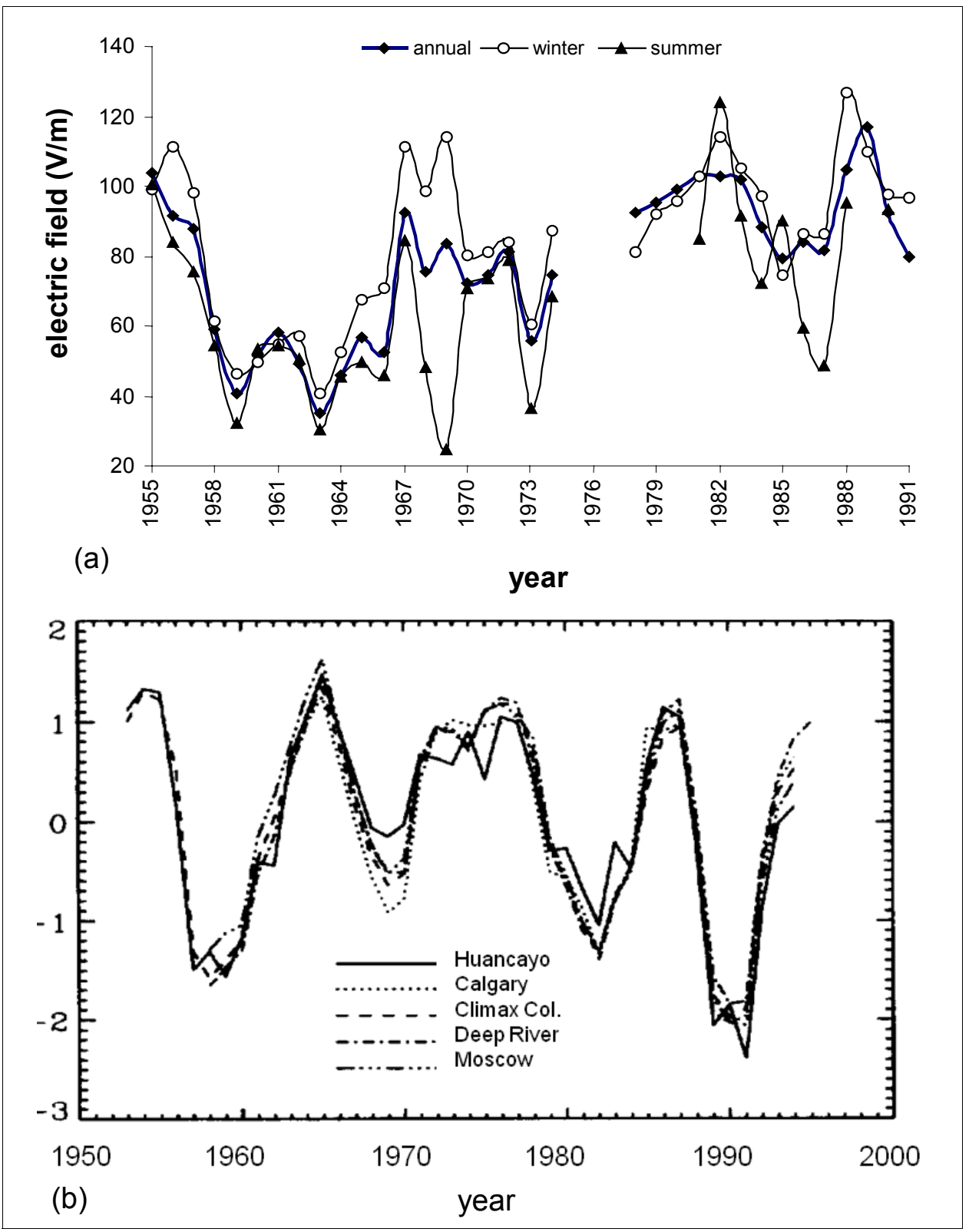

Source: Adapted from Svensmark and Friis-Christensen (1997) 
In this way, global warming is modulated by the cosmic rays effect. In the case that this effect is positive, it will reinforce the radiative imbalance, and therefore one might expect a rise in global temperature. On the other hand, if the cosmic rays effect is negative, it may completely cancel the earth's radiative imbalance pointed out by Hansen et al. (2005).

As a general remark, it must be stated that the earth's climate system is forced and modulated by an external cyclic influence that may either reinforce or mitigate local forcing of anthropogenic origin.

\section{Conclusions}

Evidence of global warming in the past few decades is accumulating, and it is virtually confirmed that it is caused by climate forcing of anthropogenic origin. We showed that external cyclic influences of cosmic origin modulate the earth's climate and may either reinforce or mitigate the 'local' terrestrial forcings. Among the external influences is cosmic radiation, whose intensity shows a cyclic variation of a period of 11 years, accompanying the 11-year cycle of solar activity. We considered how emission of particulate matter into the atmosphere might influence global lightning activity. This explanation is based on the two main ways by which the downward atmospheric electric current may reach the earth's surface: the ohmic branch and the discharge branch (lightning and corona). Atmospheric particulate matter increases the ohmic resistance and provides the conditions for an atmospheric electric breakdown, therefore leading to an increase in lightning activity. On the other hand, if cosmic radiation is at its maximum (or minimum of the solar cycle) in the 11-year cycle of solar activity, the increase in ion concentration will decrease the ohmic resistance, therefore reducing lightning activity. Also, with respect to global lightning activity, we showed why the contribution of the increase in particulate matter concentration in the atmosphere may be negligible in some years, while it will be reinforced in other years, depending on the place of the years in the cycle. We also remarked that the contribution to global warming resulting from fossil fuel burning is also modulated by the cosmic ray flux, whose influence is mediated by the variation that it promotes on the cloud cover. In this way when solar activity is close to its minimum the cosmic radiation effect on cloud cover may completely cancel the earth's radiative imbalance pointed out by Hansen et al. (2005).

\section{Acknowledgement}

This work has been supported by FCT (Portugal) under the contract PTDC/ EME-MFE/71960/2006. 


\section{References}

Bali Agreement (2007) http://www.climatesciencewatch.org/index.php/csw/details/bali_climate _declaration/.

Hansen, J., Nazarenko, L., Ruedy, R., Sato, M., Willis, J., Del Genio, A., Koch, D., et al. (2005) 'Earth's energy imbalance: confirmation and implications', Science, Vol. 308, pp.1431-1435.

Hansen, J. and Sato, M. (2004) 'Greenhouse gas growth rates', Proc. Natl. Acad. Sci., Vol. 101, pp.16109-16114.

IPPC Report (2007) IPPC, Geneva, www.ipcc.ch/ipccreports/ar4-syr.htm (accessed 2 May 2008).

Kyoto Protocol (1998) 'United Nations framework convention on climate change', http://unfccc .int/kyoto_protocol/items/3145.php.

Lyons, W.A., Nelson, T.E., Williams, E.R., Cramer, J. and Turner, T. (1998) 'Enhanced positive cloud-to-ground lightning in thunderstorms ingesting smoke', Science, Vol. 282, pp.77-81.

McGorman, D.R. and Rust, W.D. (1998) The Electrical Nature of Storms, Oxford: Oxford University Press.

Markson, R. and Price, C. (1999) 'Ionospheric potential as a proxy index for global temperatures', Atmospheric Research, Vol. 51, pp.309-314.

Serrano, C., Reis, A.H., Lucio, P. and Rosa, R. (2006) 'Influences of cosmic radiation, artificial radioactivity and aerosol concentration upon the fair-weather atmospheric electric field in Lisbon (1955-1991)', Atmospheric Research, Vol. 81, pp.236-249.

Svensmark, H. and Friis-Christensen, E. (1997) 'Variation of cosmic ray flux and cloud coverage - a missing link in solar-climate relationships', J. of Atmospheric and Solar-Terrestrial Physics, Vol. 59, No. 11, pp.1225-1232.

Williams, E., Rosenfeld, D., Madden, N., et al. (2002) 'Contrasting convective regimes over the Amazon: implications for cloud electrification', J. Geophysics. Research, Vol. 107, p.8082, doi:10.1029/2001JD000380. 